\title{
Solving Path Problems on the GPU
}

\author{
Aydın Buluça, ${ }^{\mathrm{a}, * 1}$, John R. Gilbert ${ }^{\mathrm{a}, 1}$, Ceren Budak ${ }^{\mathrm{a}}$ \\ ${ }^{a}$ Computer Science Department, University of California, Santa Barbara, CA 93106-5110
}

\begin{abstract}
We consider the computation of shortest paths on Graphic Processing Units (GPUs). The blocked recursive elimination strategy we use is applicable to a class of algorithms (such as all-pairs shortest-paths, transitive closure, and LU decomposition without pivoting) having similar data access patterns. Using the all-pairs shortest-paths problem as an example, we uncover potential gains over this class of algorithms. The impressive computational power and memory bandwidth of the GPU make it an attractive platform to run such computationally intensive algorithms. Although improvements over CPU implementations have previously been achieved for those algorithms in terms of raw speed, the utilization of the underlying computational resources was quite low. We implemented a recursively partioned all-pairs shortest-paths algorithm that harnesses the power of GPUs better than existing implementations. The alternate schedule of path computations allowed us to cast almost all operations into matrix-matrix multiplications on a semiring. Since matrix-matrix multiplication is highly optimized and has a high ratio of computation to communication, our implementation does not suffer from the premature saturation of bandwidth resources as iterative algorithms do. By increasing temporal locality, our implementation runs more than two orders of magnitude faster on an NVIDIA 8800 GPU than on an Opteron. Our work provides evidence that programmers should rethink algorithms instead of directly porting them to GPU.
\end{abstract}

Key words: All-pairs shortest paths, Gaussian elimination, graphical processing unit, semiring, matrix multiplication, graph algorithm, shortest path, linear algebra

\section{Introduction}

The massively parallel nature of GPUs makes them capable of yielding theoretically much higher GFlops rates than current state-of-the-art CPUs. GPU performance also grows much faster than CPU performance due to specialized explicit parallelism. The amount of computational power to be harvested has also attracted the high-

\footnotetext{
${ }^{*}$ Corresponding author

Email addresses: aydin@cs.ucsb.edu (Aydın Buluç), gilbert@cs.ucsb.edu (John R. Gilbert), cbudak@cs.ucsb.edu (Ceren Budak)

${ }^{1}$ The research of these authors was supported in part by the Department of Energy under award number DE-FG02-04ER25632, in part by NSF grant CNS-0709385, and in part by MIT Lincoln Laboratory under contract number 7000012980
} 
performance computing (HPC) community, and we have seen many scientific applications successfully implemented with significant performance gains on the GPU $[1,2]$.

Implementing HPC applications to run on a GPU requires significant expertise, even with the recently introduced C-like APIs such as Nvidia's Cuda platform [3]. The key to performance is to hide the data access latency by having many threads on the fly. The performance is usually fragile and requires careful craftmanship from the programmer's side. It is up to the programmer to make sure that the registers and other levels of cache are neither underutilized nor over-pressured. Several papers are devoted to the issue of achieving the right balance to get optimal performance on GPUs $[4,5]$, relying on novel programming techniques that are not necessarily intuitive to the existing HPC programmer.

An important class of algorithms with triple nested loops, which will be subsequently mentioned as Gaussian Elimination (GE) based algorithms, have very similar data access patterns. Examples include LU decomposition without pivoting, Cholesky factorization, all-pairs shortest paths (APSP), and transitive closure. The similarity among those problems has led researchers to approach them in a unified manner. For example, the Gaussian Elimination Paradigm of Chowdhury and Ramachandran provides a cache-oblivious framework for these problems [6]. In this paper, we specifically focus on the APSP problem because it usually operates on single precision floating point data, making it suitable to current generation GPUs. On the contrary, factorizations such as LU and Cholesky require double precision arithmetic that was not available on the GPUs until very recently (with AMD FireStream 9170 and Nvidia GeForce GTX 280). Even now, the double precision performance is 4-8 times slower than single precision, and the limited global memory of current generation GPUs discourage the use of double precision floating point numbers. Furthermore, numerical LU decomposition without pivoting is unstable [7] at best (it may not even exist), and pivoting strategies on the GPU are beyond the scope of this paper. Volkov and Demmel did an excellent job of implementing LU, QR, and Cholesky factorizations on the GPU, albeit in single precision [5]. It is worth noting that even though our implementation computes only the distance version of the APSP problem, it is possible to obtain the actual minimal paths, at the cost of doubling the memory requirements, by keeping a predecessor matrix.

Our two main contributions in this paper are:

1. Recursive partitioning is used as a tool to express a different schedule of path computations that allows extensive use of highly optimized matrix-matrix operations. Specifically, we use matrix multiplication on semirings as a building block for GE based algorithms. By doing so, we increase data locality, which is even more important for high performance computing on the GPU than on the CPU

2. As a proof of concept, we provide an efficient implementation of the APSP algorithm on the GPU that is up to 480x faster than our reference CPU implementation, and up to $75 \mathrm{x}$ faster than an existing GPU implementation on a similar architecture.

Locality of reference has always been an issue in algorithm design, and it will be even more important with GPUs. This is because stream processors, such as GPUs, 
achieve efficiency through locality [8]. Our work highlights the importance of recursion as a technique for automatically creating locality of reference.

As minor contributions, we give an alternate (arguably simpler) proof of correctness based on path expressions for the recursively partitioned APSP algorithm. On the GPU, we compare iterative, and recursive versions of the same algorithm and provide insights into their performance difference through micro benchmarks. Therefore, we provide evidence that Level 3 BLAS [9] routines on semirings can be used to speed up certain graph algorithms. Finally, we compare different CPUs and GPUs on their power efficiency in solving this problem.

The remainder of this paper is organized as follows. Section 2 describes the algorithms based on block-recursive elimination, starting from the well-known Gaussian Elimination procedure and using it as an analogy to explain block-recursive elimination on other algebraic structures. Most specifically, it shows how block-recursive elimination can be used to solve the all-pairs shortest-paths problem. Section 3 is devoted to GPU programming on the Cuda platform, showing difficulties and important points to achieve high performance on GPUs. Section 4 describes our implementation and evaluation strategies, and reports on the results of our experiments. Section 5 offers some concluding remarks.

\section{Algorithms Based on Block-Recursive Elimination}

Gaussian elimination is used to solve a system of linear equations $A x=b$, where $A$ is an $n \times n$ matrix of coefficients, $x$ is a vector of unknowns, and $b$ is a vector of constants. Recursive blocked LU factorization is an efficient way of performing Gaussian elimination on architectures with deep memory hierarchies [10,11]. This is mostly due to its extensive use of matrix-matrix operations (Level 3 BLAS [9]) that are optimized for the underlying architecture. Let $A$ and its factors $L$ and $U$ be partitioned as

$$
A=\left[\begin{array}{ll}
A_{11} & A_{12} \\
A_{21} & A_{22}
\end{array}\right]=\left[\begin{array}{ll}
L_{11} & \\
L_{21} & L_{22}
\end{array}\right] \cdot\left[\begin{array}{ll}
U_{11} & U_{12} \\
& U_{22}
\end{array}\right]
$$

Then, the block-recursive LU decomposition without pivoting can be written as

$$
\begin{aligned}
L_{11}, U_{11} & \leftarrow \mathrm{LU}\left(A_{11}\right) \\
U_{12} & \leftarrow L_{11} \backslash A_{12} \\
L_{21} & \leftarrow A_{21} / U_{11} \\
L_{22}, U_{22} & \leftarrow \operatorname{LU}\left(A_{22}-L_{21} U_{12}\right) .
\end{aligned}
$$

In this pseudocode, LU is the recursive call to the function itself, $\backslash$ and / denote triangular solve operations with multiple right hand sides (matrix division on the left and on the right, in MATLAB $囚$ notation).

LU factorization operates on the field of real numbers, but the same algorithm can be used to solve a number of graph problems, albeit using a different algebra. Specifically, closed semirings provide a general algebraic structure that can be used to 
solve a number of path problems on graphs [12,13]. A semiring has all the properties of a ring, except that there might be elements without an additive inverse. One practical implication is that fast matrix multiplication algorithms that use additive inverses, such as the Strassen algorithm [14] and the Coppersmith-Winograd algorithm [15], do not apply to matrices over semirings.

A closed semiring is formally denoted by $(\mathbb{S}, \oplus, \otimes, 0,1)$, where $\oplus$ and $\otimes$ are binary operations defined on the set $\mathbb{S}$ with identity elements 0 and 1 respectively. Fletcher [16] gives a complete definition of a closed semiring. Two important semirings used in this work are the Boolean semiring, formally defined as $(\{0,1\}, \vee, \wedge, 0,1)$ and the tropical semiring, formally defined as $\left(\mathbb{R}^{+}, \min ,+, \infty, 0\right)$. A closed semiring is said to be idempotent if $a \oplus a=a$ for all $a \in \mathbb{S}$. Although idempotence of the semiring is not a requirement for the solution of path problems on graphs [16], the correctness of our in-place algorithms relies on idempotence. Both the Boolean semiring and the tropical semiring are idempotent, as $\min (a, a)=a$ for all $a \in \mathbb{R}^{+}$, and $0 \vee 0=0,1 \vee 1=1$.

\subsection{The All-Pairs Shortest-Paths Problem}

The all-pairs shortest-paths (APSP) is a fundamental graph problem. Given a directed graph $G=(V, E)$ with vertices $V=\left\{v_{1}, v_{2}, \ldots, v_{n}\right\}$ and edges $E=\left\{e_{1}, e_{2}, \ldots, e_{m}\right\}$, the problem is to compute the length of the shortest path from $v_{i}$ to $v_{j}$ for all $\left(v_{i}, v_{j}\right)$ pairs. APSP corresponds to finding the matrix closure $A^{*}=\sum_{i=0}^{\infty} A^{i}=\sum_{i=0}^{n} A^{i}=$ $I \oplus A \oplus \ldots \oplus A^{n}$ on the tropical semiring. Note that we were able to avoid the problems with the infinite sum by converting it to a finite sum, because $A^{n+i}=A^{n}$ for $i>0$ in any idempotent semiring.

APSP is the focus of this paper among the set of GE based algorithms due to its practical importance and the lack of fast implementations on the GPU. All the algorithms discussed in this paper take the adjacency matrix $A$ of the graph, where $A(i, j)$ represents the length of the edge $v_{i} \rightarrow v_{j}$, as the input. They output $A^{*}$, where $A^{*}(i, j)$ represents the length of the shortest path from $v_{i}$ to $v_{j}$. Edge weights can be arbitrary (positive, negative, or zero), but we assume that there are no negative cycles in the graph. Also, the cost of staying at the same vertex is zero, i.e., $A(i, i)=0$. If not, we can delete any edge of the form $A(i, i) \neq 0$ as it will certainly not contribute to any shortest path. This is because shortest paths are simple when there are no negative cycles.

The standard algorithm for solving the APSP problem is the Floyd-Warshall (FW) algorithm. The pseudocode for the FW algorithm, in standard notation and in linear algebra notation, are given in Figures 1 and 2. It is especially well-suited for dense graphs due to its $O\left(n^{3}\right)$ complexity. It is a dynamic programming algorithm that consists of a triply nested loop similar to matrix multiplication. In fact, computing the APSP problem is computationally equivalent to computing the product of two matrices on a semiring [12]. However, the order of the loops cannot be changed arbitrarily as in the case of matrix multiplication. In the linear algebra sense, the algorithm computes the outer product of the $k$ th row and the $k$ th column, and does rank- 1 updates on the whole matrix, for $k=1,2, \ldots, n$. The order of the outer product updates cannot be changed, but one is free to compute the outer product in any order. This means that the $k$-loop should be the outermost loop, and the other loops can be freely interchanged. Although the added constraint on the order of loops hinders some of the loop- 


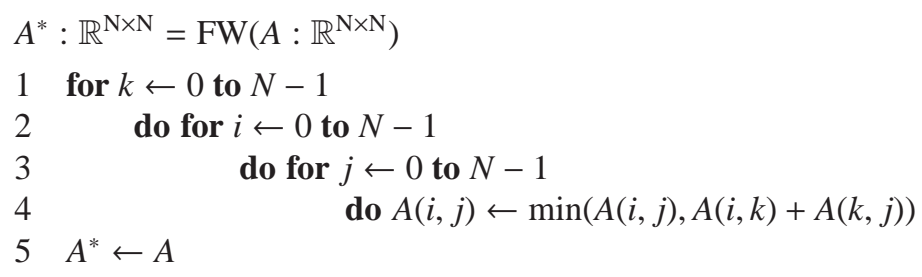

Figure 1: FW algorithm in the standard notation

$$
\begin{aligned}
& A^{*}: \mathbb{R}^{\mathrm{N} \times \mathrm{N}}=\mathrm{FW}\left(A: \mathbb{R}^{\mathrm{N} \times \mathrm{N}}\right) \\
& 1 \text { for } k \leftarrow 0 \text { to } N-1 \\
& 2 \quad \text { do } A \leftarrow A \oplus A(:, k) \otimes A(k,:) \quad \triangleright \text { Algebra on the (min, }+ \text { ) semiring } \\
& 3 A^{*} \leftarrow A
\end{aligned}
$$

Figure 2: FW algorithm in linear algebra notation

interchange optimizations that are applied to matrix multiplication, automatic program generators for the FW algorithm have been shown to provide formidable speedups [17].

For sparse graphs, Johnson's algorithm [18], which runs Dijkstra's single-source shortest paths algorithm from every vertex (after some preprocessing that lets the algorithm run on graphs having edges with negative weights), is probably the algorithm of choice for an implementation on the CPU. However, as we demonstrate in Section 4, the GE based algorithm clearly outperforms both the FW algorithm and Johnson's algorithm when implemented on the GPU.

For unweighted graphs, it is possible to embed the semiring into the ring of integers and use a fast, sub-cubic matrix multiplication algorithm such as Strassen's [14]. For an undirected and unweighted graph, Seidel [19] gives a $O(M(n) \lg n)$ algorithm, where $M(n)$ is the time to multiply two $n \times n$ matrices on the ring of integers. This elegant algorithm repeatedly squares the adjacency matrix of the graph. However, it is not currently known how to generalize Seidel's algorithm to weighted or directed graphs [20].

\subsection{Recursive In-Place APSP Algorithm}

The closure of a matrix can be computed using an algorithm similar to recursive Gaussian elimination without pivoting. It is guaranteed to terminate on a closed semiring like the tropical semiring. The only subroutine of this algorithm is matrix multiplication on a semiring. The $n$-by- $n$ adjacency matrix is recursively partitioned into four equal-sized $n / 2$-by- $n / 2$ submatrices as before; the pseudocode for the algorithm is shown in Figure 3. We use juxtaposition $(A B)$ to denote the multiplication of $A$ and $B$ on the semiring. $\beta$ is the threshold after which the algorithm performs iterative FW 


\begin{tabular}{|c|c|c|}
\hline & $\mathbb{R}^{\mathrm{N} \times \mathrm{N}}=\mathrm{APS}$ & $\mathrm{P}\left(A: \mathbb{R}^{\mathrm{N} \times \mathrm{N}}\right)$ \\
\hline 1 & if $\mathrm{N}<\beta$ & \\
\hline 2 & then $A \leftarrow$ & $\mathrm{FW}(A)$ \\
\hline 3 & & \\
\hline & & {$\left[\begin{array}{ll}A_{11} & A_{12}\end{array}\right.$} \\
\hline 4 & $A=$ & $A_{21} \quad A_{22}$ \\
\hline 5 & $A_{11}$ & $\operatorname{APSP}\left(A_{11}\right)$ \\
\hline 6 & $A_{12}$ & $-A_{11} A_{12}$ \\
\hline 7 & $A_{21}$ & $-A_{21} A_{11}$ \\
\hline 8 & $A_{22}$ & $-A_{22} \oplus A_{21} A_{12}$ \\
\hline 9 & $A_{22}$ & $-\operatorname{APSP}\left(A_{22}\right)$ \\
\hline 10 & $A_{21}$ & $-A_{22} A_{21}$ \\
\hline 11 & $A_{12}$ & $-A_{12} A_{22}$ \\
\hline 12 & $A_{11}$ & $-A_{11} \oplus A_{12} A_{21}$ \\
\hline
\end{tabular}

Figure 3: Pseudocode for recursive in-place APSP

serially instead of recursing further. The algorithm does not require $n$ to be even. If $n$ is odd, the same decomposition in (1) works with $\lfloor n / 2\rfloor$ and $\lceil n / 2\rceil$.

Both the original FW implementation given in Figures 1 and 2 as well as the recursive algorithm given in Figure 3 can be easily extended to obtain the actual minimal paths. In this case, an additional integer matrix $\Pi$ of predecessor vertices is maintained. Initially, $\Pi(i, j) \leftarrow i$ for all $i$. It is updated whenever a previously unknown path with shorter length is discovered, i.e., $\Pi(i, j) \leftarrow k$ whenever $A(i, k)+A(k, j)<A(i, j)$ during the computation. As FW and APSP are essentially performing the same computation, the discovered shortest path is guaranteed to be a path with minimal length. However, they may find different, yet equal in length, paths in the presence of multiple shortest paths for a source-destination pair. This is due to possibly different schedule of path computation.

Recursive formulations of APSP have been presented by many researchers over the years $[21,22,23]$. The connection to semiring matrix multiplication was shown by Aho et al. [12], but they did not present a complete algorithm. Ours is a modified version of the algorithm of Tiskin [23] and R-Kleene algorithm [21]. Especially, the in-place nature of the R-Kleene algorithm helped us avoid expensive global memory to global memory data copying. As the algorithm makes use of matrix multiplication as a subroutine, it has a much higher data reuse ratio while having asymptotically the same operation count.

The correctness of the recursive algorithm has been formally proven in various ways before $[21,22]$. Here we present a simpler proof based on algebraic paths. As in Aho et al. [12], we partition the set of vertices into $V_{1}=\left\{v_{1}, \ldots, v_{n / 2}\right\}$ and $V_{2}=$ $\left\{v_{n / 2+1}, \ldots, v_{n}\right\}$. Submatrix $A_{11}$ represents the edges within $V_{1}$, submatrix $A_{12}$ the edges from $V_{1}$ to $V_{2}$, submatrix $A_{21}$ the edges from $V_{2}$ to $V_{1}$, and submatrix $A_{22}$ the edges 


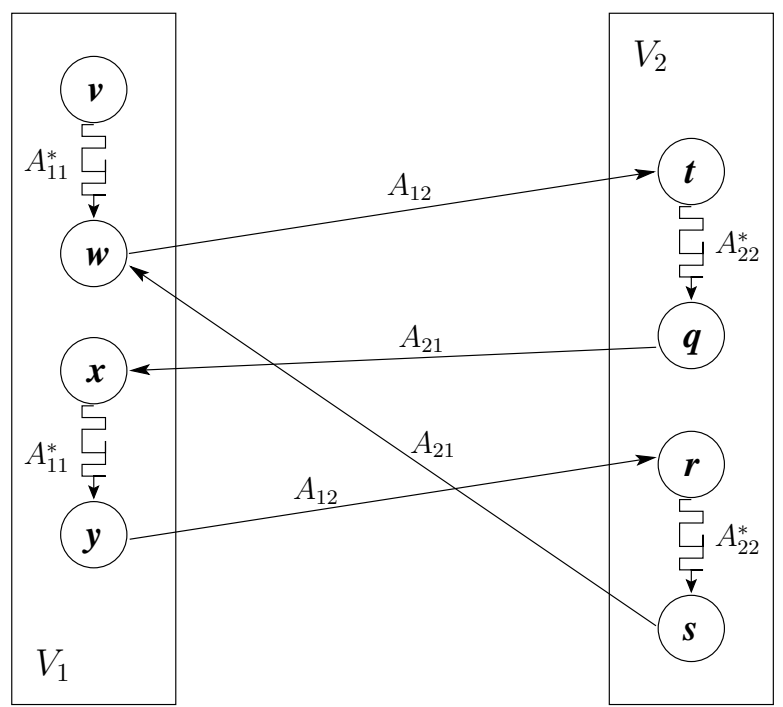

Figure 4: An example path in $A_{11}^{*}$

within $V_{2}$.

Now, consider the paths in $A_{11}^{*}$. They can either travel within $V_{1}$ only or move from $V_{1}$ to $V_{2}$ following an edge in $A_{12}$, and then come back to $V_{2}$ through an edge in $A_{21}$, possibly after traveling within $V_{2}$ for a while by following edges in $A_{22}$. The regular expression for the latter path is $A_{12} A_{22}^{*} A_{21}$. This partial path can be repeated a number of times, possibly going through different vertices each time. An example path from $v$ to $w$ is shown in Figure 4. The complete regular expression becomes

$$
A_{11}^{*}=\left(A_{11} \mid A_{12} A_{22}^{*} A_{21}\right)^{*} .
$$

On the other hand, the regular expression we get after the recursive algorithm terminates is

$$
A_{11}^{*}=A_{11}^{*} \mid\left(A_{11}^{*} A_{12}\left(A_{22} \mid A_{21} A_{11}^{*} A_{12}\right)^{*} A_{21} A_{11}^{*}\right) .
$$

These two regular expressions define the same language, hence represent the same set of paths [13]. By converting these regular expressions into deterministic finite automata (DFA), and minimizing them [24], we see that both have the same minimumstate DFA shown in Figure 5. Since the minimum-state DFA is unique for a language, this proves that the algorithm computes the correct set of paths.

It is also possible to implement this algorithm in a blocked iterative way as previously done for transitive closure [25]. The percentage of work done iteratively (without using matrix multiplication) is the same, and corresponds to the block diagonal part of the matrix. However, the multiplications in the blocked algorithm are always between matrices of size $B \times B$, where $B$ is the blocking factor. This is potentially a limiting factor on GPUs because multiplication tends to get drastically faster as matrices get bigger 


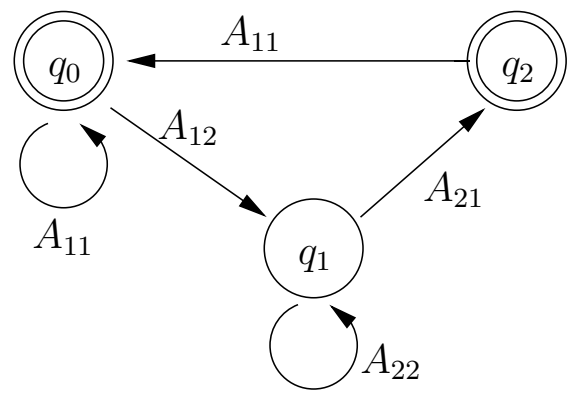

Figure 5: Minimum-state DFA for the path expressions in $A_{11}^{*}$, starting state is $q_{0}$

(less than 20 GFlops/s when $\mathrm{N}=64$ versus 200 GFlops/s when $\mathrm{N}=1024$ ) [5]. With the recursive formulation, on the other hand, more work can be done during multiplication of large matrices.

Furthermore, the recursive algorithm does fewer kernel launches than the block iterative one. The block iterative algorithm launches $O\left((N / B)^{3}\right)$ kernels for matrix multiplications and $O(N / B)$ kernels for computing closures of $B \times B$ blocks on the diagonal. On the other hand, at each level of the recursion tree, the recursive algorithm launches 6 kernels for matrix multiplications, and does 2 recursive calls. This makes a total of only $O(N / B)$ kernel launches because the height of the recursion tree is $\lg (N / B)$, and the number of kernel launches doubles at each level $(\{6,12,24, \ldots, 6(N / B)\})$. The $O\left((N / B)^{2}\right)$ factor of improvement can be quite significant, as kernel launches incur significant overhead in CUDA.

One important feature of our implementation is that it is performed in place, overwriting the input with the output without constraining the order of loops in the matrix multiplication. For the matrix multiply-add operations $A_{22} \leftarrow A_{22} \oplus A_{21} A_{12}$ and $A_{11} \leftarrow A_{11} \oplus A_{12} A_{21}$, there are no issues of correctness. However, for other multiplications of the form $B \leftarrow B A$ or $B \leftarrow A B$, the order of evaluation (whether it is an ijk loop or an kji loop) matters on a general semiring. This is because updating the output automatically updates the input, and the algorithm will now use a different input for the rest of the computation. As proved by D'Alberto and Nicolau [21], this is not a problem as long as the semiring is idempotent and $A$ is a closure. The intuition is that if the algorithm prematurely overwrites its input, this just makes the algorithm find shortest paths quicker. In other words, it speeds up the information dissemination, but the correctness is preserved thanks to idempotence.

Note that four of the six multiplications at any level of the recursion tree are of the form $B \leftarrow B A$ or $B \leftarrow A B$. In other words, they perform multiply instead of multiplyadd operations. Using $B \leftarrow B+B A$ or $B \leftarrow B+A B$ would be equally correct, but unnecessary. Remember that the cost of staying in a vertex is zero, i.e., $A(i, i)=0$. Consider $B \leftarrow A B$ : If $B$ contains a path $v_{i} \Rightarrow v_{j}$ before the operation, $A B$ generates a cost-equivalent path $v_{i} \Rightarrow v_{i} \Rightarrow v_{j}$ and safely overwrites $B$. 


\section{GPU Computing Model with CUDA}

More and more applications that traditionally run on the CPU are now being reimplemented to run on the GPU, a technique called general-purpose computing on graphics processing units (GPGPU). Both Nvidia and AMD offer programming interfaces for making GPGPU accessible to programmers who are not experts in computer graphics [26, 27]. Nvidia's Compute Unified Device Architecture (Cuda) offers a higher level C-like API, whereas AMD's Close-to-Metal (CTM) allows the programmers to access lower levels of hardware. As opposed to CTM, the Cuda platform is unified in the sense that it has no architectural division for vertex and pixel processing.

\subsection{GPU Programming}

The new generation of GPUs are basically multithreaded stream processors. They offer tremendous amounts of bandwidth and single-precision floating point arithmetic computation rates. In stream processing, a single data parallel function (kernel) is executed on a stream of data, and that is exactly how the Cuda programming model works. A Cuda program is composed of two parts: A host (CPU) code that makes kernel calls, and a device (GPU) code that actually implements the kernel. The host code is conceptually a serial C program, but the device code should be massively parallel in order to harness the power of the GPU.

The fundamental building block of Nvidia 8 and 9 series is the streaming multiprocessors (SMs), sometimes called the GPU chips. Each SM consists of 8 streaming processors (cores), but only one instruction fetch/decode unit. This implies that all 8 cores must simultaneously execute the same instruction. This is why divergence in the device code should be avoided as much as possible. The memory hierarchy consists of multiple levels. Each SM has 8192 registers and 16KB on-chip shared memory, which is as fast as registers provided that bank conflicts are avoided. A high-latency (200-300 cycles) off-chip global memory provides the main storage for the application on the GPU. Part of the off-chip memory, called the local memory, is used for storing variables that are spilled from registers.

A kernel is executed by many threads on the GPU. These threads are organized as a grid of thread blocks, which are batches of threads that can cooperate/communicate through on-chip shared memory and synchronize their execution. Each thread block is executed by only one SM, but each SM can execute multiple thread blocks simultaneously.

The main scheduling unit in Cuda is a warp, a group of 32 threads from the same thread block. All threads in a warp execute the same instruction, and execution of an arithmetic instruction for the whole warp takes 4 clock cycles. The number of active warps in a block is an important factor in tolerating global memory access latency.

\subsection{Experiences and Observations}

Some limitations exist for the device code. For example, recursion and static variables are not allowed. These limitations do not apply to the host code, as it is just a regular $\mathrm{C}$ code running on the $\mathrm{CPU}$. In fact, recursion in the host code is a powerful 
technique, since it naturally separates the recursion stack from the floating-point intensive part of the program. Although recursive divide-and-conquer algorithms are naturally cache efficient [28], they have traditionally not achieved their full performance due to the overheads associated with recursion. We do not have such a limitation with CUDA because the recursion stack, which is on the CPU, does not interfere with the kernel code on the GPU.

Code optimization on a GPU is a tedious job with many pitfalls. Performance on a GPU is often more fragile than performance on a CPU. It has been observed that small changes can cause huge effects on the performance [4]. For example, in the optimized GEMM routine of Volkov [5], each thread block is $16 \times 4$ and each thread uses 32 registers. This allows $8192 / 32=256$ threads and 256/64 $=4$ thread blocks can simultaneously be active on each SM. As there are two warps per thread block and it takes 4 cycles to execute an instruction for the whole warp, a latency of $8 \times 4=32$ cycles can be completely hidden. In the case that an extra variable is required, the compiler can either choose to spill it out to local memory and keep the register count intact, or increase the register usage per thread by one. In the latter case, the number of active thread blocks decreases to 3 . This introduces a $25 \%$ reduction in parallelism, but the former option may perform worse if the kernel has few instructions because access to a local variable will introduce one-time extra latency of 200-300 cycles. Whichever option is chosen, it is obvious that performance is fragile: by just adding one extra line, it is possible to drastically slow down the computation.

Another pitfall awaiting the programmer is bandwidth optimizations. In Cuda, peak bandwidth can only be achieved through memory coalescing, i.e., by making consecutively numbered threads access consecutive memory locations. One can heavily underutilize the GPU bandwidth by not paying attention to memory coalescing. However, the way memory coalescing works is quite counter-intuitive to a multicore programmer. Assume that one wants to scan a $16 \times N$ matrix stored in row-major order. On an SMP system with 16 cores, the most bandwidth-friendly way is to let each processor scan a different row of the matrix; in this case, each processor makes at most $N / B$ cache misses, which is optimal. On an Nvidia GPU, on the other hand, this will create multiple memory accesses per warp since these threads do not access contiguous range of memory addresses. An example with $N=8$ is shown in Figure 6. However, if the matrix were stored in column-major order, having each thread scan a different row would be optimal on an Nvidia GPU. This is because memory accesses at each step would be coalesced into a single access by the NVCC compiler [29]. Consequently, the right programming practices for achieving high bandwidth are quite different for the GPU than for traditional parallel programming.

As a result, we advocate the use of optimized primitives as much as possible on the GPU. Harris et al. provide an excellent optimized scan primitive with Cuda and encourage its use as a building block for implementing parallel algorithms on Nvidia GPUs [30]. Here, we advocate the use of matrix-matrix multiplication as an important primitive, not only for solving systems of linear equations, but also for graph computations. In terms of performance, matrix multiplication has been claimed to be unsuitable to run on GPUs due to the lack of sufficient bandwidth [31]. The new generation GPUs, however, offer a tremendous bandwidth of more than $100 \mathrm{~GB} / \mathrm{s}$. Moreover, alternate implementations that are not bandwidth bound achieved close to peak performance [5]. 


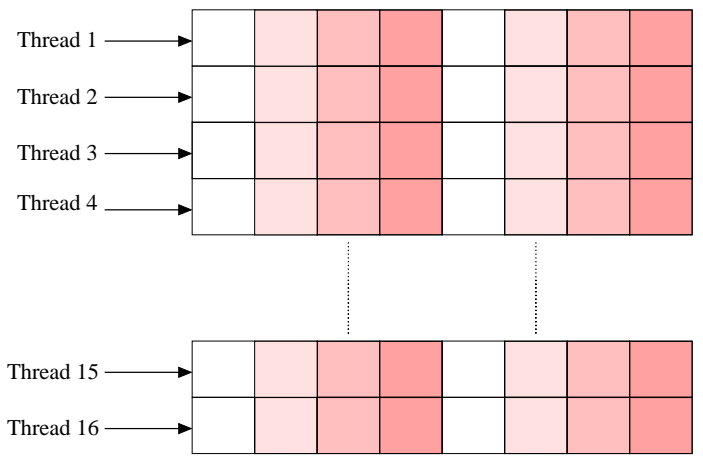

Figure 6: Stride-1 access per thread (row-major storage)

It would be wise to take advantage of such an efficient primitive whenever possible.

\section{Implementation and Experimentation}

\subsection{Experimental Platforms}

We ran our GPU code on an Nvidia GeForce 8800 Ultra with Cuda SDK 1.1 and GCC version 4.1. The graphics card driver installed in our system is Nvidia Unix x86_64 kernel module 169.09. The GeForce 8800 Ultra has 768 MB DRAM, a core clock of $612 \mathrm{MHz}$, a stream processor clock of $1.5 \mathrm{GHz}$, a memory clock of $1080 \mathrm{MHz}$, and an impressive bandwidth of $103.7 \mathrm{~GB} / \mathrm{s}$. It consists of $16 \mathrm{SMs}$, each containing 8 cores, making up a total of 128 cores. Each core can perform a multiply-add operation in a single cycle, which accounts for two floating-point operations (Flops). Therefore, it offers a peak multiply-add rate of $2 \times 1.5 \times 128=384$ GFlops/s (not counting the extra MUL operation that cores can issue only under certain circumstances).

For comparison, we ran our CPU experiments in three different settings:

1. Serial C++ code on Intel Core 2 Duo T2400 $1.83 \mathrm{Ghz}$ with 1 GB RAM running Windows XP. Two cores share a 2 MB L2 Cache.

2. Serial $\mathrm{C}++$ code on AMD Opteron $82142.2 \mathrm{Ghz}$ with $64 \mathrm{~GB}$ RAM running Linux kernel 2.6.18. Each core has a private $1 \mathrm{MB}$ L2 cache.

3. Parallel Cilk++ code on a Numa machine (Neumann) with 64 GB RAM, and 8 dual-core Opteron processors clocked at 2.2 Ghz.

\subsection{Implementation Details}

We implemented both the recursive and the iterative algorithm on the GPU using Cuda. For the recursive algorithm, we experimented with two different versions: one that uses a simple GEMM kernel, and one that uses the optimized GEMM routine of Volkov [5]. When reporting experimental results, we call the latter recursive optimized. Both recursive codes implement the same algorithm given in Figure 3. Our recursive 
Cuda code is freely available at http://gauss.cs.ucsb.edu/ aydin/apsp_cuda . html.

Our iterative APSP implementation uses a logical 2D partitioning of the whole adjacency matrix. Such a decomposition was previously employed by Jenq and Sahni on a hypercube multiprocessor [32], and found to be more effective than 1D partitioning. However, keep in mind that there is no explicit data partitioning, only a logical mapping of submatrices to thread blocks. Host code invokes the kernel $n$ times, where each thread block does a rank-1 update to its submatrix per invocation. An initial snapshot of the execution is illustrated in Figure 7 from the viewpoint of $(2,2)$ thread block.

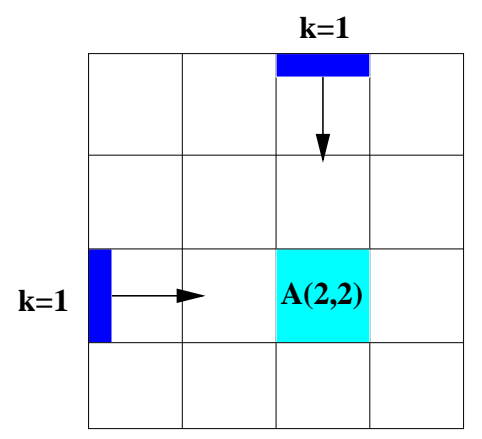

Figure 7: A shapshot from the execution of the iterative algorithm

Our serial iterative and recursive implementations run on the CPU as references. The iterative implementation is the standard implementation of FW, as shown in Figure 1. The recursive implementation is based on our recursive formulation shown in Figure 3. The recursive implemention stops the recursion when the submatrices completely fit into L1-cache to achieve better results.

Our reference parallel implementation runs on Neumann, a Numa machine with a total of 16 processor cores ( 8 dual-core $2.2 \mathrm{Ghz}$ Opterons). We used Cilk++ [33] to parallelize our code, which enabled speedups up to $15 x$.

\subsection{Performance Results}

Timings for our APSP implementations on Cuda are given in Table 1. Please note the orders of magnitude difference among implementations.

Among our reference implementations, the best CPU performance is obtained on the Intel Core 2 Duo, even though the processor had a slower clock speed than the Opteron. We attribute this difference to the superior performance of MS Visual Studio's C++ compiler. Full listings of timings obtained on two different CPUs and various compilers can be found in Appendix A. Table 2 shows the speedup of various GPU implementations with respect to the best CPU performance achieved for the given number of vertices. The results are impressive, showing up to 480x speedups over our reference CPU implementation. Using an iterative formulation, only a modest $3.1 \mathrm{x}$ speedup is achieved for relatively small inputs.

Figure 8 shows a log-log plot of running times of 5 different implementations. Iterative CPU and recursive CPU are timings obtained by our serial code running on 
Table 1: GPU timings on GeForce 8800 Ultra (in milliseconds)

\begin{tabular}{c|ccc} 
Num. of Vertices & Iterative & Recursive & Recursive Optimized \\
\hline 512 & $2.51 \times 10^{2}$ & $1.62 \times 10^{1}$ & $6.43 \times 10^{0}$ \\
1024 & $2.42 \times 10^{3}$ & $1.00 \times 10^{2}$ & $2.44 \times 10^{1}$ \\
2048 & $4.60 \times 10^{4}$ & $7.46 \times 10^{2}$ & $1.41 \times 10^{2}$ \\
4096 & $4.13 \times 10^{5}$ & $5.88 \times 10^{3}$ & $1.01 \times 10^{3}$ \\
8192 & $5.47 \times 10^{6}$ & $5.57 \times 10^{4}$ & $7.87 \times 10^{3}$
\end{tabular}

Table 2: Speedup on 8800 Ultra w.r.t. the best CPU implementation

\begin{tabular}{c|ccc} 
Num. of Vertices & Iterative & Recursive & Recursive Optimized \\
\hline 512 & 3.1 & 48.1 & 121.4 \\
1024 & 3.0 & 73.4 & 301.5 \\
2048 & 1.3 & 79.6 & 420.7 \\
4096 & 1.2 & 81.5 & 473.2 \\
8192 & 0.7 & 67.7 & 479.3
\end{tabular}

Intel Core 2 Duo. For the rest of this section, we will be referring to the recursive optimized code as our best GPU code.

Although all of the APSP algorithms scale as $n^{3}$, the observed exponent of the recursive GPU implementation turned out to be slightly different than theoretical values. To reveal that, we performed a least-squares polynomial data fit on the log-log data. The input size $(|V|)$ - running time $(t)$ relationship is of the form $t=c|V|^{n}$. This can be converted to $\lg t=\lg c+n \lg |V|$, on which we can do linear data fitting. The difference shows that in practice the performance is heavily affected by the memory traffic, not just the number of arithmetic operations performed. The observed exponents and constants are reported in Table 3.

Our best GPU implementation still outperforms the parallelized CPU code by a factor of 17-45x, even on 16 processors. Timings are listed in Table 4.

Table 3: Observed exponents and constants for the asymptotic behaviour of our APSP implementations with increasing problem size

\begin{tabular}{c|cc|ccc}
\multirow{2}{*}{$t=c|V|^{n}$} & \multicolumn{2}{|c|}{ CPU (Intel Core 2 Duo) } & \multicolumn{3}{|c}{ GPU (GeForce 8800 Ultra) } \\
\cline { 2 - 6 } & Iterative & Recursive & Iterative & Recursive & Recur. Optimized \\
\hline Exponent $(n)$ & 3.02 & 3.23 & 3.62 & 2.94 & 2.59 \\
Constant $(c)$ & $5.5 \times 10^{-6}$ & $1.4 \times 10^{-6}$ & $3.6 \times 10^{-8}$ & $1.5 \times 10^{-7}$ & $4.7 \times 10^{-7}$
\end{tabular}




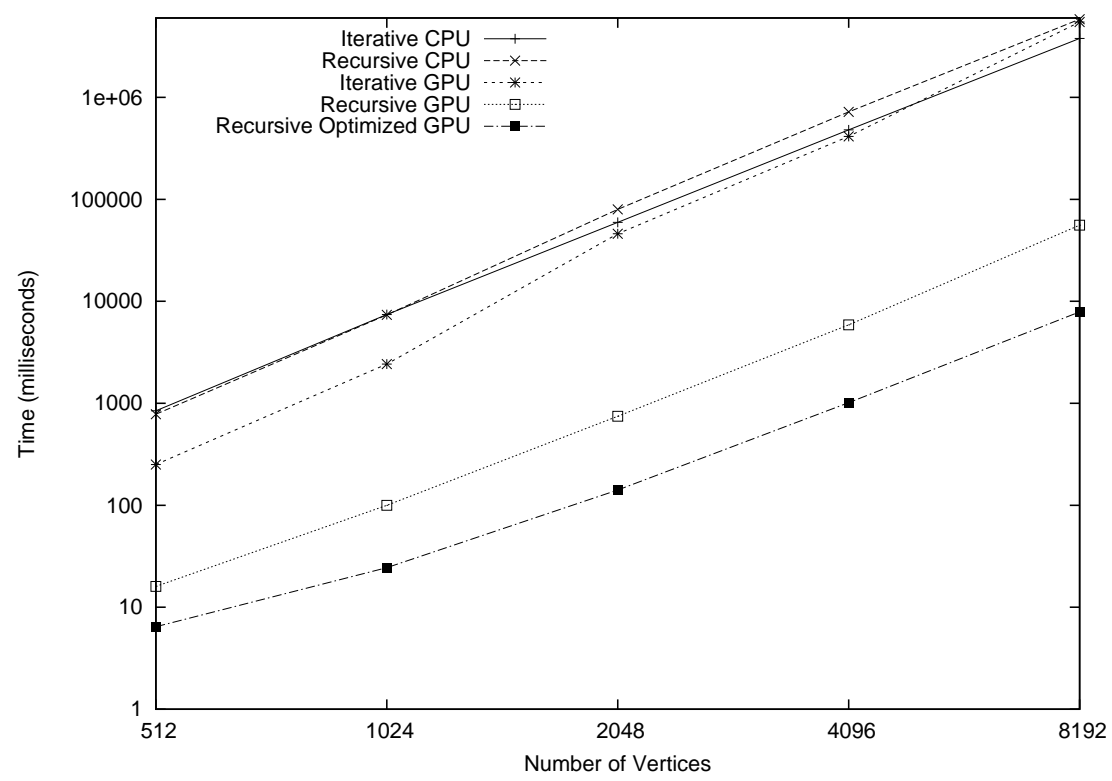

Figure 8: Log-log plot of absolute running times

\subsection{Comparison with Earlier Performance Results}

We compare the performance of our code with two previously reported results. One is an automatically generated, highly optimized serial program running on a $3.6 \mathrm{Ghz}$ Pentium 4 CPU [17]. The other is due to Harish and Narayanan on a GPU platform very similar to ours [34]. Our GeForce 8800 Ultra is slightly faster than the GeForce 8800 GTX used by Harish and Narayanan, so we underclocked our GPU to allow a direct comparison in terms of absolute values.

On the GPU, Harish and Narayanan implemented two variants of APSP: one that uses the FW algorithm and one that runs Dijkstra's single source shortest paths (SSSP) algorithm for every vertex. For sparse graphs with $m=O(n)$, the latter is theoretically

Table 4: Performance comparison of our best (optimized recursive) GPU implementation with parallel Cilk++ code running on Neumann, using all 16 cores

\begin{tabular}{c|ccc} 
Num. of Vertices & Best GPU (secs) & Parallel CPU (secs) & GPU Speedup \\
\hline 512 & 0.00643 & 0.113 & $17.5 \times$ \\
1024 & 0.0244 & 0.708 & $29 \times$ \\
2048 & 0.141 & 5.146 & $36.5 \times$ \\
4096 & 1.01 & 40.36 & $40 \times$ \\
8192 & 7.87 & 354.9 & $45 \times$
\end{tabular}




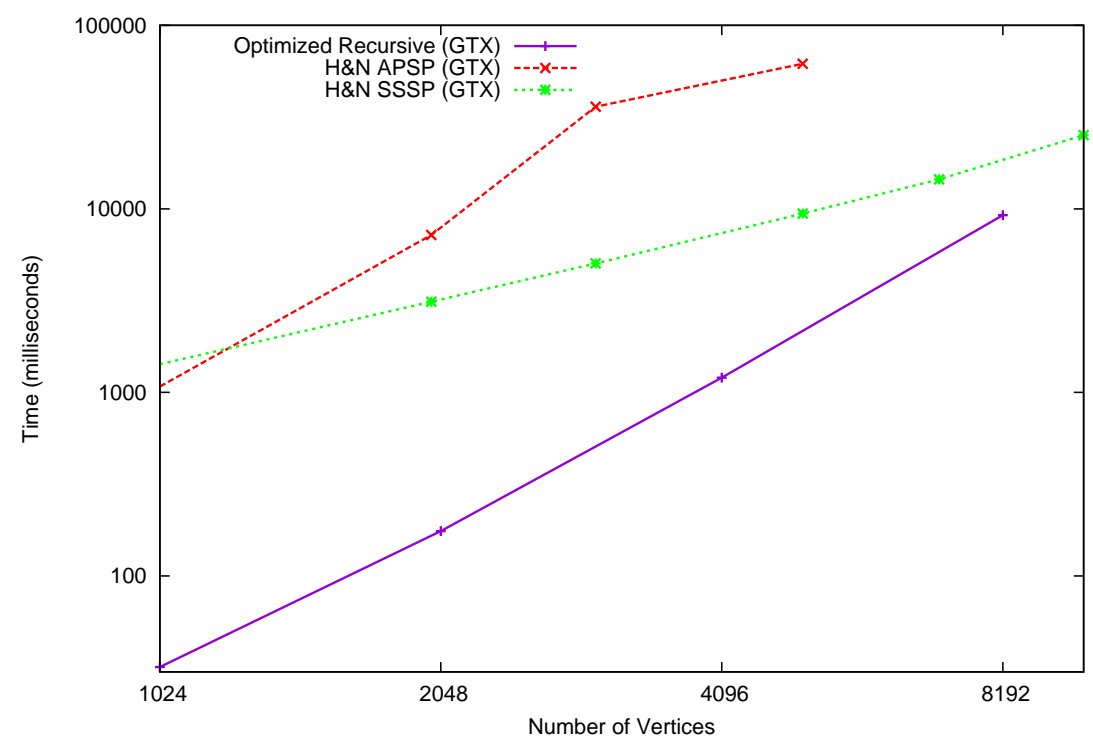

Figure 9: Comparison of different GPU implementations on 8800 GTX settings

faster than both the FW algorithm and our recursive formulation in the classical RAM model of computation [12]. It runs in $O\left(n^{2} \lg n+n m\right)$ time using Fibonacci heaps [35].

As seen in Figure 9, our recursive implementation significantly outperforms both their FW implementation (H\&N APSP) and Dijkstra based implementation (H\&N SSSP) when implemented on a GPU. The running times for the H\&N SSSP code are observed for randomly generated Erdős-Rényi graphs with an average vertex degree of 6 . The running times of the other two implementations are not sensitive to sparsity. When timing our algorithm, we underclocked our GPU's clocks down to the speed of 8800 GTX for a head-to-head comparison. Due to the adjacency matrix representation, our algorithm runs on graphs of at most 8192 vertices. Therefore, the H\&N SSSP implementation is currently more favorable for large sparse graphs, although it lags behind in terms of raw speed. We plan to implement an out-of-core version of our algorithm for larger graphs. The asymptotic behavior (the slope of the curve) of the H\&N SSSP implementation is also favorable but the test graphs used by them are extremely sparse, which helps the SSSP implementation whose complexity depends on the sparsity of the input.

The performance results for our iterative algorithm, given in Section 4.3, agree with the $2 \mathrm{x}-3 \mathrm{x}$ speedup over a CPU implementation achieved by H\&N APSP. That implementation was also limited to 4096 vertices, while ours extends to 8192 with only a slowdown over the CPU implementation. Our best APSP code is faster than H\&N APSP by a factor of 35-75x.

Comparing our results with the timings reported by Han et al. for the optimized code obtained using their auto generation tool Spiral [17], we also see significant 
speedups achieved by our best (optimized recursive) GPU implementation. Our comparisons are against their vectorized code (typically 4-5x faster than scalar code), and we see speedups up to 28x against Pentium 4, and 42x against Athlon 64. A detailed comparison can be found in Table 5. Those results also show that the GPU implementation scales better with increasing problem size, because the speedup we get over Spiral increases as the problem size increases.

Table 5: Comparisons of our best GPU implementation with the timings reported for Han et al. 's auto generation tool Spiral

\begin{tabular}{c|ccc|cc}
\multirow{2}{*}{ Num. of Vertices } & \multicolumn{3}{|c|}{ GFlops/s } & \multicolumn{2}{c}{ Speedup of GeForce } \\
\cline { 2 - 6 } & GeForce 8800 & Pentium 4 & Athlon 64 & Pentium 4 & Athlon 64 \\
\hline 512 & 38.6 & 5.08 & 3.17 & $7.6 \mathrm{x}$ & $12.2 \mathrm{x}$ \\
1024 & 82.0 & 5.00 & 2.77 & $16.4 \mathrm{x}$ & $29.6 \mathrm{x}$ \\
2048 & 113.5 & 4.78 & 2.73 & $23.7 \mathrm{x}$ & $41.6 \mathrm{x}$ \\
4096 & 126.7 & 4.47 & 2.96 & $28.3 \mathrm{x}$ & $42.8 \mathrm{x}$
\end{tabular}

\subsection{Scalability and Resource Usage}

In this section, we try to identify the bottlenecks in our implementation in terms of resource usage and scalability. By using the NVIDIA Coolbits utility, we tweaked the frequencies of both the GPU core clock and the memory clock. The results reveal that our recursive implementation is not limited by the memory bandwidth to global GPU DRAM. For this implementation, the timings and GFlops/s rates with different clock rates are given in Table 6. When the memory clock is fixed, the slowdown of the computation closely tracks the slowdown of the GPU core clock (0-50\% with increments of $12.5 \%$ ). On the other hand, when the GPU core clock is fixed, little slowdown is observed when we underclock the memory clock. Coolbits reported the default clock speeds of 8800 Ultra as $648 \mathrm{Mhz}$ for cores, and $1152 \mathrm{Mhz}$ for memory, which are slightly different than the values reported in NVIDIA factsheets.

The peak rate observed was 130 GFlops/s for $|V|=8192$, compared to the theoretical peak of 384 GFlops. However, the theoretical peak counts 2 Flops for each fused multiply-add operation, which is not available on the tropical semiring our algorithm operates on. Therefore, the actual theoretical peak in the absence of fused multiplyadd operations is 192 GFlops. Our implementation achieves more than $67 \%$ of that arithmetic peak rate for APSP.

The iterative implementation, on the other hand, is observed to be completely bandwidth bound. Even when the GPU cores are underclocked to half, no slowdown was observed. Underclocking the memory to half, however, slowed down the computation by exactly a factor of two. Exact timings can be seen in Figure 7. We conclude that the iterative formulation is putting too much stress on GPU memory bandwidth, consequently not harnessing the available computation power of the GPU. This is indeed expected, because the iterative formulation accesses $O\left(n^{2}\right)$ data and does $O\left(n^{2}\right)$ work in every iteration. The recursive algorithm, on the other hand, does almost all of its work 
Table 6: Scalability of our optimized recursive GPU implementation. We tweaked core and memory clock rates using Coolbits.

\begin{tabular}{l|ccccc}
$|V|=4096$ & GPU Clock & Memory Clock & Time $(\mathrm{ms})$ & GFlops/s & Slowdown $(\%)$ \\
\hline Default values & 648 & 1152 & 1028.3 & 124.4 & - \\
\hline & 567 & 1152 & 1190.8 & 107.5 & 13.6 \\
Memory & 486 & 1152 & 1362.9 & 93.9 & 24.5 \\
clock fixed at & 405 & 1152 & 1673.1 & 76.5 & 38.5 \\
$1152 \mathrm{Mhz}$ & 324 & 1152 & 2093.7 & 61.1 & 50.8 \\
\hline & 648 & 1008 & 1036.2 & 123.5 & 0.7 \\
GPU core & 648 & 864 & 1047.3 & 122.2 & 1.8 \\
clock fixed at & 648 & 720 & 1096 & 116.8 & 6.1 \\
648 Mhz & 648 & 576 & 1124.9 & 113.8 & 8.5
\end{tabular}

Table 7: Scalability of our iterative GPU implementation. We tweaked core and memory clock rates using Coolbits.

\begin{tabular}{c|cccc}
$|V|=4096$ & GPU Clock & Memory Clock & Time $(\mathrm{ms})$ & Slowdown $(\%)$ \\
\hline Default values & 648 & 1152 & 417611.4 & - \\
Core clock halved & 324 & 1152 & 418845.7 & 0.3 \\
Memory clock halved & 648 & 576 & 856689.7 & 51.2
\end{tabular}

in matrix multiplications, which access $O\left(n^{2}\right)$ data for doing $O\left(n^{3}\right)$ work. Therefore, it clearly has better locality of reference.

As it was not possible to disable a subset of GPU cores in the NVIDIA 8800, we do not report any scalability results with increasing number of processors.

\subsection{Power and Economic Efficiency}

Power efficiency is becoming an important consideration when comparing different architectures [36]. The Green500 list ranks supercomputers according to their Flops/Watts $\times$ sec (or Flops/Joule) ratio. In this section, we compare the power efficiency of different architures for the APSP problem, using power specs of the manufacturer's equipment (in Thermal Watts)

Nvidia reports a peak power consumption of 175 Watts for its GeForce 8800 Ultra video card. Our dual-core Opteron (model number 8214) is reported to consume a peak power of 95 Watts, but we are using only a single core of it during serial computation. The machines used in the reported timings of automatically tuned CPU implementations are Pentium 4 (model number 560) and Athlon 64 (model 4000+). They consume 115 and 89 Watts, respectively. The Intel Core Duo T2400, the most power efficient CPU in this comparison, has a maximum power consumption of only 31 Watts even when both cores are active.

This comparative study should be considered very preliminary, because we are not running the same code in every architecture. The GPU code is assumed to use $175+$ 
Table 8: Efficiency comparison of different architectures (running various codes), values in MFlops/Watts $\times$ sec (or equivalently MFlops/Joule)

\begin{tabular}{c|c|cc|c|c}
\multirow{2}{*}{$|V|$} & Nvidia GPU & Athlon & Pentium 4 & Core 2 Duo & Neumann (Opteron) \\
\cline { 2 - 6 } & Best Cuda code & \multicolumn{2}{|c|}{ Spiral Code } & Reference FW & Cilk++ $(\mathrm{p}=16)$ \\
\hline 512 & 173 & 35.6 & 44.1 & 19.1 & 2.9 \\
1024 & 368 & 31.1 & 43.7 & 17.4 & 3.7 \\
2048 & 510 & 30.6 & 41.5 & 17.3 & 4.1 \\
4096 & 569 & 33.2 & 38.8 & 17.2 & 4.2
\end{tabular}

$95 / 2=222.5$ Watts as it also uses one of the CPU cores to assist the computation. This is also a rough estimate as it is likely that when one core is idle, the whole processor's power consumption is more than half of its maximum. However, our rationale is that it is possible to use the other core to perform the same computation on a different input.

The results, outlined in Table 8, show that the Nvidia Cuda implementation is not only powerful, but also efficient. The closest competitor is the auto generated Spiral [17] code that runs on Pentium 4. Note that Pentium 4 is not a particularly power efficient processor. Therefore, it is plausible that an auto generated code on more power efficient hardware would get closer to the efficiency of the GPU. A couple of factors contribute to the inefficiency of Neumann. The most important one being that the Opterons we use are not high-efficiency (HE) versions, but rather high-performance Opterons. A single Opteron core in Neumann consumes more than three times the power that is consumed by Core 2 Duo, while still giving worse performance in this particular problem.

Looking at the timings are listed in Table 4, the economic efficiency of the GPU is also clear. At the time of writing, the processors of our 8-way Opteron server is priced about 7x the price of Nvidia GPUs we have been using. Given that the GPU implementation runs about 17-45x faster, we see Flops/Dollar ratio of the GPU is up to $119-315 x$ better than an 8 -way server. These statements are by no means conclusive as they are based on APSP performance only.

\section{Conclusions and Future Work}

We have considered the efficient implementation of Gaussian elimination based algorithms on the GPU. Choosing the right algorithm that efficiently maps to the underlying hardware has always been important in high-performance computing. Our work shows that it is even more important when the hardware in question is a GPU. Our proof-of-concept implementation runs more than two orders of magnitude faster than a simple porting of the most popular algorithm to the GPU. The key to performance was to choose an algorithm that has good locality of reference and makes the most use of optimized kernels.

We made extensive comparisons with our reference implementations on single processor and shared memory multiprocessor systems, as well as with previously reported 
results obtained on various CPUs and GPUs. Future work includes identifying and implementing crucial kernels that are likely to speed up a large class of applications. Specifically, we are working on implementing an efficient sparse matrix-matrix multiplication algorithm on the GPU, which is to be used as a building block for many graph algorithms [37, 38].

\section{Acknowledgments}

We acknowledge the kind permission of Charles Leiserson and CilkArts to use an alpha release of the Cilk++ language. We also thank P.J.Narayanan and Pawan Harish for providing us the exact timings from their experiments. Sivan Toledo helped us improve the presentation of the paper with various comments. Thanks to Fenglin Liao and Arda Atali for their help during the initial implementation on Cuda. Finally, we would like to thank three anonymous reviewers for their constructive comments that improved the presentation.

\section{References}

[1] J. Bolz, I. Farmer, E. Grinspun, P. Schrder, Sparse matrix solvers on the GPU: Conjugate gradients and multigrid, ACM Transactions on Graphics 22 (3) (2003) 917-924.

[2] S. Sengupta, M. Harris, Y. Zhang, J. D. Owens, Scan primitives for GPU computing, in: Graphics Hardware 2007, ACM, 2007, pp. 97-106.

[3] E. Lindholm, J. Nickolls, S. F. Oberman, J. Montrym, Nvidia Tesla: A unified graphics and computing architecture, IEEE Micro 28 (2) (2008) 39-55.

[4] S. Ryoo, C. I. Rodrigues, S. S. Baghsorkhi, S. S. Stone, D. B. Kirk, W.-M. W. Hwu, Optimization principles and application performance evaluation of a multithreaded GPU using CUDA, in: PPoPP '08: Proceedings of the 13th ACM SIGPLAN Symposium on Principles and Practice of Parallel Programming, ACM, New York, NY, USA, 2008, pp. 73-82.

[5] V. Volkov, J. Demmel, LU, QR and Cholesky Factorizations using Vector Capabilities of GPUs, Tech. Rep. UCB/EECS-2008-49, EECS Department, University of California, Berkeley (May 2008).

[6] R. A. Chowdhury, V. Ramachandran, Cache-oblivious dynamic programming, in: SODA '06: Proceedings of the Seventeenth Annual ACM-SIAM Symposium on Discrete Algorithm, ACM, New York, NY, USA, 2006, pp. 591-600.

[7] G. H. Golub, C. F. Van Loan, Matrix Computations, The Johns Hopkins University Press, 1996.

[8] W. J. Dally, Keynote address: "Stream programming : Parallel processing made simple", in: ICPP '08: Proc. of the Intl. Conf. on Parallel Processing, IEEE Computer Society, 2008. 
[9] J. J. Dongarra, J. Du Croz, S. Hammarling, I. S. Duff, A set of level 3 basic linear algebra subprograms, ACM Transactions on Mathematical Software 16 (1) (1990) 1-17.

[10] E. Elmroth, F. Gustavson, I. Jonsson, B. Kågström, Recursive blocked algorithms and hybrid data structures for dense matrix library software, SIAM Review 46 (1) (2004) 3-45.

[11] S. Toledo, Locality of reference in LU decomposition with partial pivoting, SIAM Journal of Matrix Analysis and Applications 18 (4) (1997) 1065-1081.

[12] A. V. Aho, J. E. Hopcroft, J. D. Ullman, The Design and Analysis of Computer Algorithms, Addison-Wesley Longman, Boston, MA, USA, 1974.

[13] R. E. Tarjan, A unified approach to path problems, Journal of the ACM 28 (3) (1981) 577-593.

[14] V. Strassen, Gaussian elimination is not optimal, Numerical Math. 13 (1969) 354356.

[15] D. Coppersmith, S. Winograd, Matrix multiplication via arithmetic progressions, in: STOC '87: Proceedings of the Nineteenth Annual ACM Conference on Theory of Computing, ACM Press, New York, NY, USA, 1987, pp. 1-6.

[16] J. G. Fletcher, A more general algorithm for computing closed semiring costs between vertices of a directed graph, Communications of the ACM 23 (6) (1980) 350-351.

[17] S.-C. Han, F. Franchetti, M. Püschel, Program generation for the all-pairs shortest path problem, in: PACT '06: Proceedings of the 15th International Conference on Parallel Architectures and Compilation techniques, ACM, New York, 2006, pp. 222-232.

[18] D. B. Johnson, Efficient algorithms for shortest paths in sparse networks, Journal of the ACM 24 (1) (1977) 1-13.

[19] R. Seidel, On the all-pairs-shortest-path problem in unweighted undirected graphs, Journal of Computer and System Sciences 51 (3) (1995) 400-403.

[20] U. Zwick, Exact and approximate distances in graphs - a survey, in: ESA '01: Proceedings of the 9th Annual European Symposium on Algorithms, SpringerVerlag, 2001, pp. 33-48.

[21] P. D'Alberto, A. Nicolau, R-Kleene: A high-performance divide-and-conquer algorithm for the all-pair shortest path for densely connected networks, Algorithmica 47 (2) (2007) 203-213.

[22] J.-S. Park, M. Penner, V. K. Prasanna, Optimizing graph algorithms for improved cache performance, IEEE Transactions on Parallel and Distributed Systems 15 (9) (2004) 769-782. 
[23] A. Tiskin, Synchronisation-efficient parallel all-pairs shortest paths computation (work in progress), http://www.dcs.warwick.ac.uk/ tiskin/pub/2004/ apsp.ps (2004).

[24] J. E. Hopcroft, R. Motwani, J. D. Ullman, Introduction to Automata Theory, Languages, and Computation (2nd Edition), Addison Wesley, 2000.

[25] J. D. Ullman, M. Yannakakis, The input/output complexity of transitive closure, Annals of Mathematics and Artificial Intelligence 3 (2-4) (1991) 331-360.

[26] AMD Stream Computing, http://ati.amd.com/technology/ streamcomputing.

[27] NVIDIA CUDA, http://www.nvidia.com/cuda.

[28] F. G. Gustavson, Recursion leads to automatic variable blocking for dense linearalgebra algorithms, IBM J. Res. Dev. 41 (6) (1997) 737-756.

[29] NVIDIA, CUDA Programming Guide 1.1, http://developer.download. nvidia.com/compute/cuda/1_1/NVIDIA_CUDA_Programming_Guide_1. 1.pdf (2007).

[30] M. Harris, S. Sengupta, J. D. Owens, Parallel prefix sum (scan) with CUDA, in: H. Nguyen (Ed.), GPU Gems 3, Addison Wesley, 2007.

[31] K. Fatahalian, J. Sugerman, P. Hanrahan, Understanding the efficiency of GPU algorithms for matrix-matrix multiplication, in: HWWS '04: Proceedings of the ACM SIGGRAPH/EUROGRAPHICS Conference on Graphics Hardware, ACM, New York, 2004, pp. 133-137.

[32] J. Jenq, S. Sahni, All pairs shortest paths on a hypercube multiprocessor, in: ICPP '87: Proc. of the Intl. Conf. on Parallel Processing, 1987, pp. 713-716.

[33] Cilk Arts, Inc., Burlington, MA, Cilk++ Programmer's Guide, available from http://www.cilk.com/ (2009).

[34] P. Harish, P.J.Narayanan, Accelerating large graph algorithms on the GPU using CUDA, in: International Conference on High Performance Computing (HiPC 2007), 2007.

[35] M. L. Fredman, R. E. Tarjan, Fibonacci heaps and their uses in improved network optimization algorithms, Journal of the ACM 34 (3) (1987) 596-615.

[36] W. Feng, X. Feng, R. Ge, Green supercomputing comes of age, IT Professional 10 (1) (2008) 17-23.

[37] A. Buluç, J. R. Gilbert, Challenges and advances in parallel sparse matrix-matrix multiplication, in: ICPP '08: Proc. of the Intl. Conf. on Parallel Processing, Portland, Oregon, USA, 2008, pp. 503-510. 
[38] A. Buluç, J. R. Gilbert, On the representation and multiplication of hypersparse matrices, in: IEEE International Parallel and Distributed Processing Symposium (IPDPS 2008), 2008.

[39] J. G. Siek, L.-Q. Lee, A. Lumsdaine, The Boost Graph Library User Guide and Reference Manual (With CD-ROM), Addison-Wesley Professional, 2001. 


\section{Appendix A. Additional Timing Results}

Table 9 shows the timings obtained on Intel Core 2 Duo, using MS Visual Studio 2003's C++ compiler. For small inputs $(|V| \leq 1024)$, the recursive implementation performs better due to its cache friendliness. For larger inputs, however, the overhead of recursion starts to dominate the running time. We have also experimented with the Boost Graph Library's Floyd-Warshall implementation [39] but found it to be consistently slower than our implementations. This might be due to the overheads coming from the genericity of Boost. Therefore, we excluded its running times from our plots in the main text.

Table 9: Serial timings on Intel Core 2 Duo (in milliseconds)

\begin{tabular}{c|ccc} 
Num. of Vertices & Iterative & Recursive & Boost \\
\hline 512 & $8.43 \times 10^{2}$ & $7.81 \times 10^{2}$ & $1.37 \times 10^{3}$ \\
1024 & $7.40 \times 10^{3}$ & $7.35 \times 10^{3}$ & $1.16 \times 10^{4}$ \\
2048 & $5.94 \times 10^{4}$ & $7.98 \times 10^{4}$ & $9.19 \times 10^{4}$ \\
4096 & $4.79 \times 10^{5}$ & $7.20 \times 10^{5}$ & $7.27 \times 10^{5}$ \\
8192 & $3.77 \times 10^{6}$ & $5.82 \times 10^{6}$ & N.A.
\end{tabular}

In Table 10, we list the performance of our reference implementations, compiled both with GCC and Intel $\mathrm{C} / \mathrm{C}++$ compiler version 9.1 (ICC). Although Intel's compiler consistently outperformed GCC, its performance still lags behind the performance achieved by MS Visual Studio on Intel.

Table 10: Serial timings on Opteron (in milliseconds)

\begin{tabular}{c|cc|cc}
\multirow{2}{*}{ Num. of Vertices } & \multicolumn{2}{|c|}{ Iterative } & \multicolumn{2}{c}{ Recursive } \\
\cline { 2 - 5 } & GCC & ICC & GCC & ICC \\
\hline 512 & $1.30 \times 10^{3}$ & $9.90 \times 10^{2}$ & $1.60 \times 10^{3}$ & $1.14 \times 10^{3}$ \\
1024 & $1.07 \times 10^{4}$ & $8.31 \times 10^{3}$ & $1.34 \times 10^{4}$ & $9.74 \times 10^{3}$ \\
2048 & $8.41 \times 10^{4}$ & $6.41 \times 10^{4}$ & $1.32 \times 10^{5}$ & $1.03 \times 10^{5}$ \\
4096 & $6.66 \times 10^{5}$ & $5.03 \times 10^{5}$ & $1.24 \times 10^{6}$ & $1.00 \times 10^{6}$ \\
8192 & N.A. & $3.94 \times 10^{6}$ & N.A. & $1.58 \times 10^{7}$
\end{tabular}

